\title{
Effects of candesartan cilexetil on carotid remodeling in hypertensive diabetic patients: the MITEC study
}

\author{
JP Baguet' \\ R Asmar ${ }^{2}$ \\ $P$ Valensi ${ }^{3}$ \\ S Nisse-Durgeat ${ }^{4}$ \\ JM Mallion' \\ 'Clinique de Cardiologie, $\mathrm{CHU}$ \\ de Grenoble, Grenoble, France; \\ ${ }^{2}$ Institut CardioVasculaire, Paris, \\ France; ${ }^{3}$ Service d'Endocrinologie- \\ Diabétologie-Nutrition, $\mathrm{CHU}$ Jean \\ Verdier, AP-HP, Bondy, France; \\ ${ }^{4}$ Laboratoires Takeda, Puteaux, France
}

Correspondence: $\mathrm{Pr}$ Jean-Philippe Baguet Clinique de Cardiologie, $\mathrm{CHU}$ de Grenoble - BP 217, 38043 Grenoble Cedex 09, France

Tel +33 (0) 476768480

$\mathrm{Fax}+33(0) 476765559$

Email jpbaguet@chu-grenoble.fr

\begin{abstract}
In hypertension and diabetes, early structural changes of the arterial wall precede or support atherosclerosis. There is evidence that some antihypertensive drugs exert an antiatherosclerotic effect. Over 36 months, we investigated the effect of candesartan cilexetil (CC) on the common carotid intima-media thickness (IMT) vs amlodipine besylate (AML) in patients with type 2 diabetes and mild to moderate essential hypertension. After a 4-week wash-out period, 209 patients were randomized to either CC $8 \mathrm{mg}$ or AML $5 \mathrm{mg}$ once daily for a minimum of 1 month, after which, if BP was not normalized, the dosage was doubled, followed by the addition of hydrochlorothiazide $12.5 \mathrm{mg}$ if necessary. No significant differences were observed between the two groups for change in IMT at M12 ( $-0.001 \mathrm{vs}-0.027 \mathrm{~mm} / \mathrm{year}$ for CC and AML respectively, $p=0.425)$, at M24 ( $-0.033 \mathrm{vs}-0.019 \mathrm{~mm}$ per year respectively, $\mathrm{p}=0.442$ ), and at the last visit $(-0.016 \mathrm{vs}-0.039 \mathrm{~mm}$ per year respectively, $\mathrm{p}=0.549)$. Within the group, comparisons did not show a significant difference in changes in IMT from baseline to the three visits. At the last visit, IMT regression was observed in $52.2 \%$ of patients receiving CC and in $51.3 \%$ of those receiving AML $(\mathrm{p}=0.908)$. The augmentation in carotid lumen diameter from baseline was statistically greater in the AML group at the last visit $(p=0.034)$. BP variations during the study were similar in the two groups. The results of this study show that $\mathrm{CC}$ and AML treatments may alter identically the natural progression of carotid IMT in hypertensive type 2 diabetic patients.
\end{abstract}

Keywords: hypertension, diabetes, carotid, intima-media thickness, candesartan, amlodipine

\section{Introduction}

Hypertension is present in more than $50 \%$ of patients with diabetes. Hypertension and diabetes are two cardiovascular risk factors leading to atherosclerosis, which is the source of most cardiovascular complications. ${ }^{1}$ In hypertension and diabetes, early structural changes of the arterial wall, with or without hypertrophy, precede or support atherosclerosis. Early lesions of atherosclerosis can be detected by noninvasive examination, such as ultrasonography of the carotid artery, and are powerful predictors of future cardiovascular events. ${ }^{2,3}$ This method has been used in several therapeutic trials. ${ }^{4}$ Carotid ultrasonography is a painless, reliable and reproducible examination which makes it possible to measure the intima-media thickness at different points on the carotid artery (CIMT). Detection of the early phases of atherosclerosis is essential from a diagnostic and therapeutic point of view, and improves the knowledge of pathophysiological mechanisms. Improving the control of hypertension and diabetes can reduce the progressive plaque formation associated with arterial wall thickening.

There is evidence that antihypertensive drugs, or at least some classes of antihypertensive drugs, in particular calcium antagonists and angiotensin converting enzyme inhibitors, exert an antiatherosclerotic effect that is partly independent of the blood pressure (BP)-lowering effect of these drugs. ${ }^{5,6}$ 
Candesartan cilexetil (CC) is a selective AT1 subtype angiotensin II receptor antagonist (ARB). Clinical studies in over 9000 patients proved candesartan to be an effective and well-tolerated treatment for hypertension. Meta-analyses of 6 placebo-controlled European studies showed that the average reduction in diastolic BP (DBP) is approximately $5.6 \mathrm{mmHg}(95 \%$ confidence interval [CI]: $4.3-7 \mathrm{mmHg})$ at a dose of CC $8 \mathrm{mg}$ per day. ${ }^{7}$ The average reduction in DBP observed when the dose is doubled to $16 \mathrm{mg}$ is approximately $7.1 \mathrm{mmHg}$ (95\% CI: $5.5-8.6 \mathrm{mmHg}$ ). The average fall in DBP when the $8 \mathrm{mg}$ dose is taken in association with hydrochlorothiazide (HCTZ) is approximately $7.9 \mathrm{mmHg}$. ${ }^{7}$

Amlodipine besylate (AML) is a second-generation dihydropyridine calcium antagonist, effective in the treatment of hypertension. The beneficial action of AML on the CIMT was highlighted in several studies. ${ }^{8-10}$ The mechanism of AML in slowing down CIMT progression seems to be due not only to reduction of the pulse pressure (PP) but also to a direct antiatherogenic effect of AML.

The objective of this study was to evaluate the effect of CC compared with AML on the CIMT using ultrasound methods in patients with type 2 (non-insulin dependent) diabetes and mild to moderate essential hypertension.

\section{Materials and methods Study design}

The design features have been previously reported ${ }^{11}$ and are summarized as follows. MITEC (Media Intima Thickness Evaluation with Candesartan cilexetil) was a multicenter, randomized, double-blind, active controlled, and parallelgroup study conducted in France, in hypertensive type 2 diabetic patients. The objective was to evaluate the effect of $\mathrm{CC}$ in the progression of CIMT over 36 months in comparison with AML.

The study was conducted in hospital departments or private physicians' offices. Investigators participating in the study were cardiologists or general practitioners. The trial was carried out in accordance with the Helsinki Declaration, and the French law for biomedical research, and was approved by the local Research Ethics Committee of Grenoble (France). All subjects gave written informed consent. The study coordinating committee could stop the study prematurely if necessary.

\section{Study population}

Ambulatory men or women (40-74 years old) were randomized if there was evidence of mild to moderate (140/90 $\leq \mathrm{BP}<180 / 110 \mathrm{mmHg})$, essential, non-complicated hypertension with type 2 diabetes treated by diet and/or oral hypoglycemic agents, stable for at least 3 months. Hypertension, defined according to ESH-ESC 2003 guidelines $^{12}$ had either been recently diagnosed and not previously treated, or previously unsuccessfully treated in terms of efficacy or tolerability. In addition, patients had to disply: a common CIMT, measured by ultrasonography, of $\geq 0.6$ and $\leq 1.2 \mathrm{~mm}$ (measured in 5 places by ultrasonography callipers); total cholesterol $<2.5 \mathrm{~g} / \mathrm{L}$, triglycerides $<4 \mathrm{~g} / \mathrm{L}$ and low-density lipoprotein (LDL) cholesterol $<1.6 \mathrm{~g} / \mathrm{L}$ (under lipid lowering drugs or not); $\mathrm{HbA} 1 \mathrm{c} \leq 10 \%$; compliance to the single-blind placebo treatment during run-in of between $80 \%$ and $120 \%$ (evaluated by counting returned capsules). Dyslipidemia was demonstrated by elevation of total cholesterol, LDL-cholesterol or triglyceride concentrations, or a decrease in high-density lipoprotein (HDL) cholesterol concentration in the plasma.

Patients were not included if they had: type 1 (insulindependent) diabetes, type 2 diabetes requiring insulin, secondary diabetes, secondary or malignant hypertension, severe hypertension at rest (systolic BP $[\mathrm{SBP}] \geq 180 \mathrm{mmHg}$ and/or DPB $\geq 110 \mathrm{mmHg}$ ), congestive heart failure, myocardial infarction or stroke during the previous 6 months, cardiac arrhythmia, previous carotid surgery or stenting, significant carotid stenosis, non-validated carotid ultrasonography, severe renal insufficiency, abnormal hepatic function tests.

\section{Interventions}

After a 4-week placebo wash-out period, patients were randomized to receive either CC $8 \mathrm{mg}$ or AML $5 \mathrm{mg}$ once daily, encapsulated for blinding purposes. Randomization was computer generated and balanced by the center. If BP was not normalized at any follow-up visit (from M1 to M33), ie, $\mathrm{SBP} \geq 130 \mathrm{mmHg}$ or $\mathrm{DBP} \geq 85 \mathrm{mmHg}$, the treatment could be doubled. If BP was still not normalized, HCTZ $12.5 \mathrm{mg}$ (half a $25 \mathrm{mg}$ oral tablet) had to be administered daily. After 3 months of uncontrolled BP, patients were excluded from the study due to efficacy failure. No return to a former posology after adjustment was allowed.

\section{Carotid ultrasonography}

Carotid ultrasonography was made at baseline and yearly thereafter (M12, M24, and M36). For each center, baseline and follow-up studies were performed in a standard fashion by a single specialist physician who was specially trained to do the prescribed study examination. B-mode ultrasonography was performed on a single ultrasound machine with a $7.5 \mathrm{MHz}$ sector scanner probe (axial and lateral resolution $<0.25 \mathrm{~mm}$ ) as previously described. ${ }^{13}$ 
Both common carotid arteries were studied consecutively in the long axis with probe incidence allowing good quality images. A zoom was used to define a zone of interest of $20 \mathrm{~mm}$ in length (stretching from 10 to $30 \mathrm{~mm}$ above the carotid bifurcation). A good image was defined by the presence of two hyper-echogenic lines, separated by a hypoechogenic zone, from the posterior artery wall. The IMT was defined as the distance separating the most internal parts of these lines (B mode image of intima plus media thickness) and the luminal diameter by the distance between the bloodintima interfaces on the anterior and posterior walls. ${ }^{14}$ All images were stored electronically and analyzed by specific software (TIMC laboratory, CHU Grenoble, France). IMT and diameter were measured semi-automatically on areas free of atheroma. The mean number of measurements of common carotid IMT at each site was $122 \pm 15$. The value of CIMT for any subject was the mean value for the two common carotid arteries. An independent observer who was blinded to treatment group and trained in the interpretation of CIMT images performed off-line analysis of B-mode ultrasound images. Regression in CIMT between two visits was defined by a reduction in IMT whereas progression was noted in case of an increase in IMT.

\section{Blood pressure assessment}

At each visit, BP was measured just before carotid 1 using a mercury sphygmomanometer according to ESH-ESC guidelines. ${ }^{12} \mathrm{BP}$ was measured in the sitting position after 5 minutes of rest, 3 times at 1-minute intervals, the average being used to define office values. BP was measured once more after 1 minute in the standing position.

\section{Safety assessments}

All adverse events (AEs) reported by the patients or observed by the investigator were recorded in the case report form at each visit whatever the causal relation to treatment. AEs have been reported to the appropriate drug licensing authority and to the drug manufacturer. An ECG and biological measurements were performed prior to each visit. Biological measurements were performed after an overnight fast of at least 12 hours and included: plasma creatinine, creatinine clearance (Cockroft formula) sodium, potassium, glucose, hemoglobin $A_{1 c}$ $\left(\mathrm{HbA}_{1 \mathrm{c}}\right)$, total cholesterol, HDL cholesterol, triglycerides and LDL cholesterol (calculated by the Friedwald formula).

\section{Statistical analysis}

Sample size calculation assumed a 3-year difference in CIMT changes between treatments groups of $0.05 \mathrm{~mm}$ with a standard deviation of $0.10 \mathrm{~mm}$. With two sided $5 \%$ significance, 95\% power, and 30\% drop-out rate, a total sample size of 220 patients was aimed to be recruited into the study.

The efficacy criteria were evaluated in the per-protocol population. Due to the small number of patients reaching M36, the changes in CIMT were analyzed at M12, M24, and at the last available visit.

Changes in CIMT for each treatment were compared using Student's t-test; a Wilcoxon test was performed if the data were not normally distributed. Changes in the other quantitative variables were analyzed similarly. The qualitative variables were analyzed using a chi-squared test or the degree of significance calculated using Fisher's exact test. Pearson's coefficient was used to analyze the correlation between the CIMT values and other variables as follows: demographic and clinical characteristics, BP measurements, and biological laboratory values classically considered as cardiovascular risk factors, at M0, M12, M24 and at the last visit; $\mathrm{p}<0.05$ was deemed to indicate significance.

\section{Results \\ Patients}

A total of 254 patients were selected from 131 sites and entered in a 4-week, single blind, placebo run-in/wash-out period (Figure 1). Among these, 209 were randomized and allocated to CC $(n=100)$ or AML $(n=109)$. During the follow-up, 98 patients discontinued the study prematurely (43\% in CC group vs 50.5\% in AML group). AML patients exhibited more AEs related to treatment than CC patients, which led to early study discontinuation (Figure 1). Furthermore, AML patients (21.2\%) discontinued more frequently for uncontrolled hypertension than $\mathrm{CC}$ patients $(12 \%)$. The study design did not allow an increase in doses of treatment if patients were not controlled with $16 \mathrm{mg}$ of CC or $10 \mathrm{mg}$ of AML, with or without $12.5 \mathrm{mg} \mathrm{HCTZ}$ association. This led to a significant number of withdrawals for lack of efficacy (16.7\%), and a significant number of patients (46.8\%) did not complete the planned 36-month treatment period. It was the decision of the study coordinating committee and sponsor to stop the study prematurely.

\section{Characteristics of study population}

\section{at baseline}

The baseline characteristics are given in Table 1. Patients, principally male $(63.6 \%)$, were $59.7 \pm 8.5$ years old. There was good comparability of baseline characteristics between groups, except for the presence of dyslipidemia $(\mathrm{p}=0.027)$ which was more frequent in the AML group. 


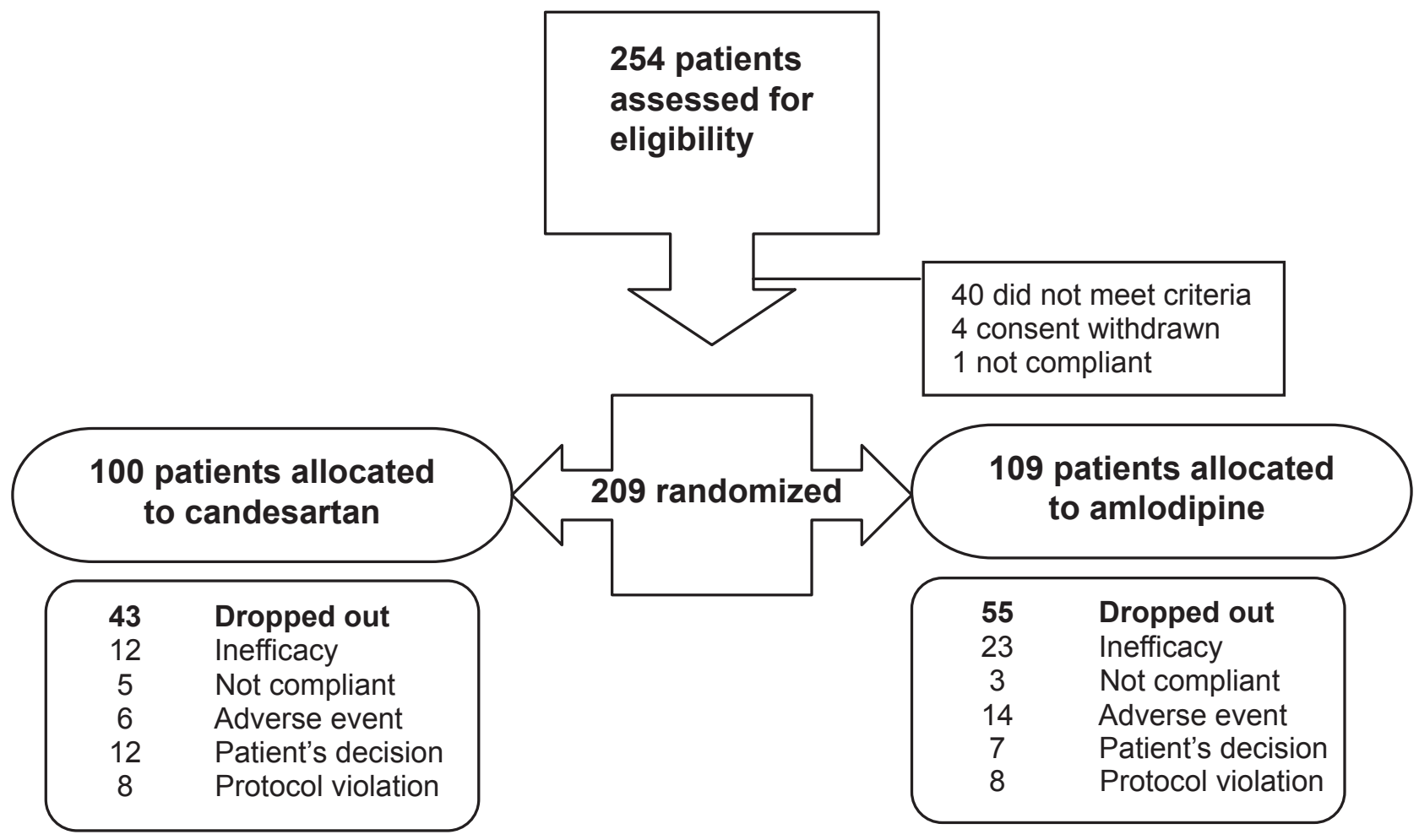

Figure I Flow of patients through the trial.

The median time since diagnosis for hypertension and diabetes was 5.9 years $(0-41)$ and 4.7 years $(0-36)$, respectively. Hypertension was treated for $75.6 \%$ of patients, and the majority $(57.9 \%)$ received more than 3 treatments. Diabetes was treated by an appropriate diet $(21.2 \%)$, or a diet in association with antidiabetic treatment (63.4\%), or only with antidiabetic treatment (14.1\%). Oral antidiabetic treatments were mainly sulfonylureas and biguanides.

The CIMT at baseline was positively correlated to $\mathrm{HbAlc}$ $(p=0.032)$, creatinine $(p=0.014)$ and age $(p<0.001)$, and negatively to creatinine clearance $(\mathrm{p}=0.005)$.

\section{Changes in the carotid wall CIMT}

During the first year, the CIMT $\mathrm{T}_{\text {median }}$ decrease was $-0.001 \mathrm{~mm}$ per year and $-0.027 \mathrm{~mm}$ per year for $\mathrm{CC}$ and $\mathrm{AML}$ regimen treatment respectively $(p=0.425)$. During the second year of treatment, the CIMT ${ }_{\text {median }}$ decrease was $-0.033 \mathrm{~mm} /$ year and $-0.019 \mathrm{~mm}$ per year for $\mathrm{CC}$ and $\mathrm{AML}$ regimen treatment respectively $(\mathrm{p}=0.442)$.

A reduction in the $\mathrm{CIMT}_{\text {median }}$ was observed at the last visit in the CC group and the AML group, with no statistical difference between treatments $(\mathrm{p}=0.549)$.

Within the group, comparisons did not show a statistically significant difference in the changes in CIMT from baseline to M12, M24, or to the last visit. There was no difference observed between treatment groups in any of the M12, M24, and last visit CIMT measurements (Table 2). At the last visit, CIMT regression was observed in $56.5 \%$ of patients receiving $\mathrm{CC}$ and $59.0 \%$ of those receiving AML ( $\mathrm{p}=0.820$ ), compared with the baseline visit.

\section{Carotid lumen diameter}

Carotid lumen diameter changed slightly with $\mathrm{CC}$ and $\mathrm{AML}$ regimen treatment. The increase in carotid lumen diameter from baseline was statistically greater in the AML group at the last visit $(p=0.034)$. However, there were no significant changes from baseline within each treatment group (Table 2).

\section{Cross-sectional area}

A trend towards a decrease in cross-sectional area was observed. However, the SD values indicate important dispersion. The changes in cross-sectional area, at M12, at M24, and at the last visit from baseline were not significant within each treatment group (Table 2, Figure 3 ) and did not differ significantly between groups $(\mathrm{p}=0.089)$.

Treatment-related changes in blood pressure, heart rate, and biological parameters BP normalization were seen from the third month of treatment in $51 / 88$ patients (58\%) from the CC group and $49 / 93$ patients (51.6\%) from 
Table I Characteristics of the study population at baseline (intent to treat population)

\begin{tabular}{|c|c|c|c|}
\hline & Candesartan $N=100$ & Amlodipine $\mathbf{N}=109$ & $\mathbf{p}$ \\
\hline Male sex, n (\%) & $60(60.0)$ & $73(67.0)$ & 0.295 \\
\hline Age, years & $59.7 \pm 8.6$ & $59.7 \pm 8.4$ & 0.755 \\
\hline $\mathrm{BMI}^{\mathrm{a}}, \mathrm{kg} / \mathrm{m}^{2}$ & $31.0 \pm 5.6$ & $30.3 \pm 4.8$ & 0.540 \\
\hline Glucose, g/L & $1.6 \pm 0.5$ & $1.5 \pm 0.4$ & 0.774 \\
\hline $\mathrm{HbAlc}, \%$ & $7.1 \pm 1.5$ & $7.0 \pm I .1$ & 0.778 \\
\hline Creatinine, mg/L & $8.8 \pm 1.6$ & $9.1 \pm 2.3$ & 0.564 \\
\hline Creatinine clearance $^{\mathrm{a}}, \mathrm{mL} / \mathrm{min}$ & $108 \pm 37$ & $107 \pm 34$ & 0.902 \\
\hline \multicolumn{4}{|l|}{ Serum lipids, g/L } \\
\hline Total cholesterol & $2.0 \pm 0.3$ & $2.1 \pm 0.4$ & 0.763 \\
\hline LDL cholesterol & $1.2 \pm 0.3$ & $1.2 \pm 0.3$ & 0.794 \\
\hline HDL cholesterol & $0.5 \pm 0.1$ & $0.5 \pm 0.1$ & 0.151 \\
\hline Triglycerides & $1.6 \pm 1.3$ & $1.7 \pm 1.0$ & 0.329 \\
\hline Lipid disorders, \% & $52(52.0)$ & $73(67.0)$ & 0.027 \\
\hline \multicolumn{4}{|l|}{$\mathrm{BP}, \mathrm{mmHg}$} \\
\hline Systolic BP & $156 \pm 12$ & $156 \pm 11$ & 0.820 \\
\hline Diastolic BP & $91 \pm 8$ & $92 \pm 8$ & 0.283 \\
\hline Pulse pressure & $65 \pm 12$ & $64 \pm 11$ & 0.247 \\
\hline Heart rate, bpm & $74 \pm 9$ & $73 \pm 9$ & 0.358 \\
\hline
\end{tabular}

Note: Results are given as mean \pm SD.

aCalculated by the Cockcroft formula.

Abbreviations: BMI, body mass index; BP, blood pressure.

AML, and progressively increased in both groups: $54.8 \%$ (40/73 patients) and 57.7\% (41/71 patients) at M12, and $61.5 \%$ (24/39 patients) and 65.8\% (25/38 patients) at M24 (Figure 2).

At the final visit, office BP decreased markedly $(\mathrm{p}<0.001)$ and equally in both treatment groups: $-25 /-14 /-11 \mathrm{mmHg}$ and $-25 /-16 /-10 \mathrm{mmHg}$ for SBP, DBP, and PP values in $\mathrm{CC}(\mathrm{n}=36)$ and AML $(\mathrm{n}=33)$ groups, respectively. No significant changes were observed in heart rate.

Serum lipids, glucose, and HbA1c did not show differences between baseline and the last visit. Creatinine increased by $0.6 \mathrm{mg} / \mathrm{L}$ and $1.2 \mathrm{mg} / \mathrm{L}$ in the $\mathrm{CC}$ and $\mathrm{AML}$ groups, respectively. Creatinine clearance fell significantly by $-8 \mathrm{~mL} / \mathrm{min}(\mathrm{p}<0.001)$ and $-14 \mathrm{~mL} / \mathrm{min}(\mathrm{p}=0.002)$ in the $\mathrm{CC}$ and $\mathrm{AML}$ groups, respectively. No correlations were found between changes in CIMT and BP measurements.

\section{Treatment adaptation and concomitant treatments}

At M12, 17 (23\%), 18 (25\%), and 38 (52\%) of the patients treated with $\mathrm{CC}(\mathrm{n}=73$ evaluated) received a low-dose monotherapy, a double dose, or add-on HCTZ, respectively. Of the patients treated with AML $(\mathrm{n}=71$ evaluated), $22(31 \%)$,
24 (34\%), and 25 (35\%) received low-dose monotherapy, a double dose, or add-on HCTZ, respectively.

At M24, of the patients treated with CC $(n=39$ evaluated), 7 (18\%), $12(31 \%)$, and 20 (51\%) received a low-dose monotherapy, a double dose or add-on HCTZ, respectively, while of the patients treated by AML $(n=38$ evaluated), 14 (37\%), 12 (31.5\%), and 12 (31.5\%) received low-dose monotherapy, a double dose, or add-on HCTZ, respectively.

Anti-diabetic agents and lipid-lowering treatments were given during the treatment period. At M12, 106/146 patients $(72 \%)$ received an antidiabetic agent and $72 / 147$ patients $(49 \%)$ received a lipid-lowering treatment. At M24, of the 78 patients evaluated, 59 (75.6\%) received an antidiabetic and $38(48.7 \%)$ patients received a lipidlowering agent.

\section{Safety}

Significantly more AEs related to the study drug occurred in the AML group than in the CC group (32.1\% vs $13.0 \%$ respectively, $\mathrm{p}=0.001)$. The number of serious AEs was not statistically different between the two groups. During the study, 21 patients (10\%) withdrew due to adverse events, 
$\bullet \mathrm{CC} \backsim \mathrm{AML}$

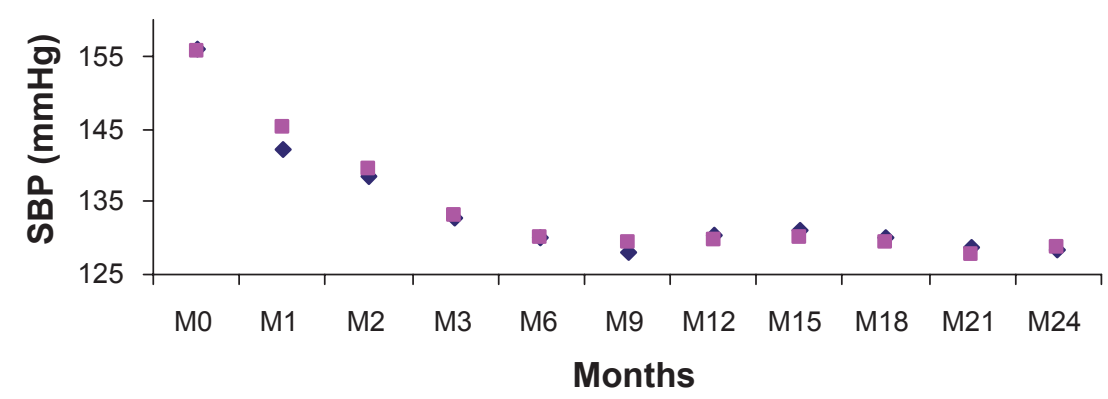

B

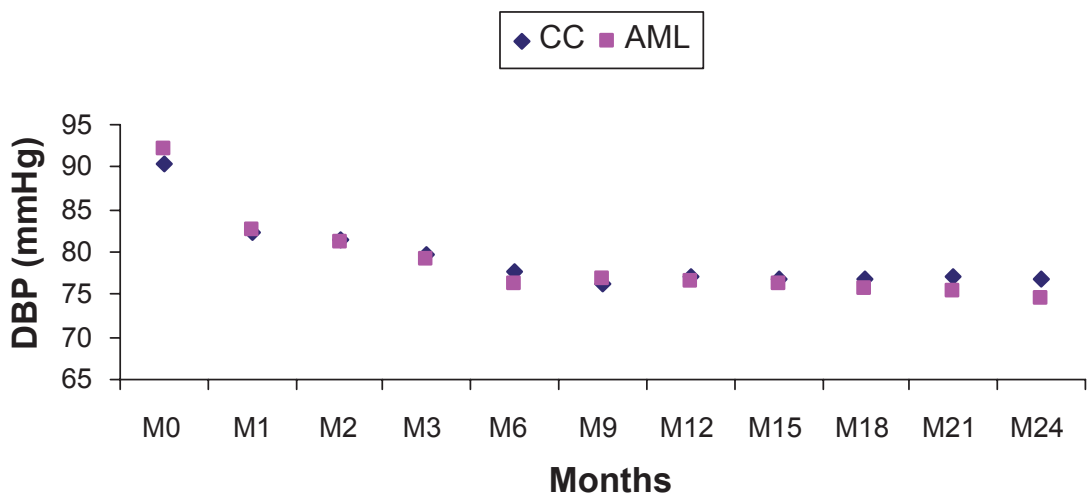

Figure 2 Evolution of clinic SBP A) and DBP B) values from baseline (M0) to M24 in hypertensive diabetic patients treated by candesartan cilexetil (CC) or amlodipine (AML). Patients evaluated in CC group: 89(M0), 86(MI), 8I(M2), 79(M3), 7I (M6), 7I (M9), 64(MI2), 62(MI5), 59(MI8), 46(M2I), 36(M24).

Patients evaluated in AML group: 93(M0), 89(MI), 86(M2), 82(M3), 64(M6), 62(M9), 60(MI2), 52(MI5), 48(MI8), 37(M2I), 33(M24).

5 patients from the CC group and 16 patients from the AML group. No patient died during the study.

\section{Discussion}

This is the first study to date, to our knowledge, to assess the efficacy of CC on CIMT progression in diabetic patients. The principal results of the MITEC study showed that antihypertensive treatment with $\mathrm{CC}$ decreased CIMT in diabetic patients to the same extent as treatment with AML. Looking at the change in CIMT from baseline to the last visit, the median values fell with both $\mathrm{CC}$ and AML regimen treatments. This fall was not statistically significant between or within groups, but represents a tendency. Our results failed to support the hypothesis that CC was superior to AML but showed a similar effect of both treatments in diabetic patients.

It is important to note that MITEC was prematurely stopped due to the number of patients discontinuing the study mainly due to lack of antihypertensive efficacy
(CC 12\% and AML 21\%). The number of patients evaluated in the per-protocol population was smaller than required in the study design to achieve a statistically significant conclusion. Premature withdrawals can be explained by several factors. A total of $57.9 \%$ of randomized patients required at least 3 anti-hypertensive drugs. Among these patients, replacement of the treatment with a placebo during the first month and the impossibility of intensifying the antihypertensive treatment, after up-titration and addition of HCTZ $12.5 \mathrm{mg}$, are likely to have influenced the decision to stop trial participation. If not controlled, the progression of BP levels in these diabetic patients would have created worsening conditions for the vessels and the atherosclerotic lesions with increasing arterial stiffness. A previous study with similar doses of AML,${ }^{10}$ which demonstrated a significant decrease in CIMT $(-0.048 \mathrm{~mm})$ compared with lisinopril $(-0.027 \mathrm{~mm})$, allowed doxazosin and bendrofluazide as second- and third-line therapies, in case of unacceptable control. 
Altogether, the rigid study therapy regimen and the reduced final analysis population contributed to a lack of statistically significant findings for CIMT. However, a downward trend in CIMT values deserves further investigation into the role of $\mathrm{CC}$ in reducing CIMT in hypertensive patients with diabetes. Supporting our results, a recent study conducted in hypertensive patients demonstrated the role of CC in reducing CIMT after 52 weeks of treatment. ${ }^{15}$

It was previously described that healthy subjects show a progression in CIMT values of 0.007 to $0.008 \mathrm{~mm}$ per year. ${ }^{16,17}$ In the study by Hanefeld et al ${ }^{18}$ patients with glucose intolerance and treated with placebo had an annual progression rate of $0.013 \mathrm{~mm}$ per year, which is almost twice that of healthy subjects. Patients with type 2 diabetes exhibited a higher annual increase in CIMT $(+0.02 \mathrm{~mm}$ per year). ${ }^{19}$ The effects of some antidiabetic agents, arcabose vs placebo, ${ }^{18}$ repaglidine vs glyburide,${ }^{20}$ or the insulin sensitizer rosiglitazone vs placebo, ${ }^{21}$ on CIMT progression have been principally tested in diabetic patients while studying oral antidiabetics. Other studies tested the effects on CIMT progression in hypertensive or coronary artery disease patients by using antihypertensive treatments: AML vs placebo, ${ }^{8}$ AML vs lisinopril, ${ }^{10}$ ramipril vs placebo, ${ }^{19}$ nifedipine vs co-amilozide, ${ }^{22}$ or lacidipine vs atenolol. ${ }^{23}$ Pitt et a ${ }^{8}$ described the competence of AML in patients with coronary artery disease for the first time and showed a significant reduction of CIMT vs placebo. The SECURE study ${ }^{19}$ examined the effect of $10 \mathrm{mg}$ ramipril, an angiotensin converting enzyme inhibitor, on CIMT during a 4-year follow-up where annual progression was reduced to $0.018 \mathrm{~mm}$ with ramipril, vs $0.022 \mathrm{~mm}(\mathrm{p}=0.033)$ in the placebo group.

Our patients combined both hypertension and diabetes. The search for a correlation between CIMT values and various parameters showed only a correlation with age, a main correlation factor observed in other studies. ${ }^{24}$ The metabolic parameters were well controlled and stable throughout the study. In the patients who remained in the study with CIMT measurements available, both treatments, $\mathrm{CC}$ and $\mathrm{AML}$, were effective in managing hypertension, as assessed by the fall in measured BP. Although BP was not associated with CIMT at baseline, the fall in BP seems to play an important role in decreasing CIMT values. However, our data do not exclude a significant role for the decrease in $\mathrm{PP}$, particularly local carotid $\mathrm{PP},{ }^{25}$ or antiatherogenic effects with both drugs.

Preliminary evidence suggests that a decrease in BP is associated with diminished wall thickness. ${ }^{10}$ It is not known if all classes of antihypertensive agents have similar protective 


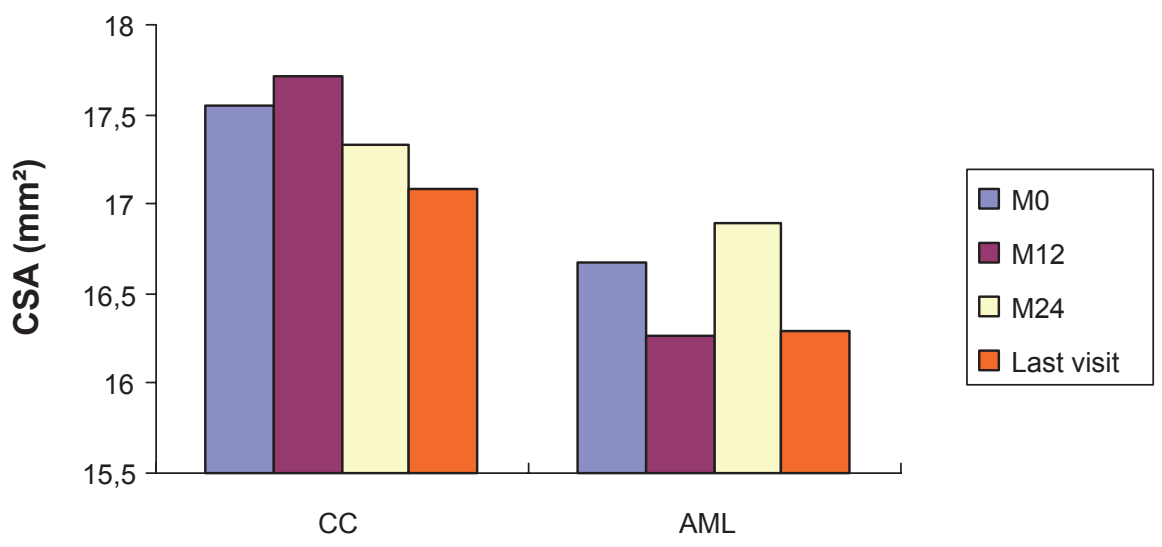

Figure 3 Cross-sectional area (CSA) values at baseline (M0), MI2, M24, and at last visit in hypertensive diabetic patients treated by candesartan cilexetil (CC) or amlodipine (AML). No significant change within each treatment group.

effects. Comparing our results with those in the literature, the regression for CIMT values in $56.5 \%$ of diabetic patients treated with $\mathrm{CC}$ is an exciting and promising result. Further research must be conducted with a large number of patients to confirm these results.

It has been shown that CIMT progression rates measured at the internal carotid artery rather than the common carotid artery detect greater absolute changes in CIMT and are better correlated with cardiovascular risk factors. The results of a 3-year study of a community population showed that mean CIMT progression was significantly greater at the internal carotid $(0.032 \pm 0.109 \mathrm{~mm}$ per year $)$ than the carotid bifurcation $(0.023 \pm 0.108 \mathrm{~mm}$ per year $)$ and common carotid artery $(0.001 \pm 0.040 \mathrm{~mm}$ per year $)(\mathrm{p}<0.001){ }^{26}$ Furthermore, only the internal CIMT correlated significantly with baseline risk factors (age, male gender, hypertension, diabetes, and smoking). Observing these site-specific differences may be important in future studies testing the effects of antihypertensive drugs on vascular remodelling and CIMT.

The changes in cross-sectional area showed a similar trend in both treatment groups. The lumen diameter was enlarged under AML but not under CC, principally due to the higher vasodilatation effect of AML than with CC.

The changes in CIMT observed in the present study and the disparate vascular remodeling seen in the two treatments groups may not be attributable only to the direct action of the drugs on the arterial wall, but also to differences in local hemodynamics, and shear and tensile stresses within the carotid artery. AML, a member of the long-acting highly lipophylic dihydropyridines, has a marked inhibitory influence on the oxygen-free radicals involved in lipid peroxidation and has been shown to prevent experimental atherosclerosis.

ARBs have been shown to cause outward remodeling in resistance arteries, ${ }^{27}$ which may be related to vasodilation. ${ }^{28}$
A previous study ${ }^{29}$ reported that ARB losartan reduced CIMT to a similar extent to atenolol. However, a small substudy of the LIFE trial showed a significant decrease in CIMT with losartan therapy, but not with atenolol therapy, after 3 years of treatment..$^{30}$ Another study ${ }^{31}$ reported that an 8 -week treatment with irbesartan decreased radial artery wall thickness in comparison with placebo but did not affect CIMT or lumen diameter. Factors influencing the carotid lumen diameter are not well understood. ${ }^{15,27,28,30,31}$ Ariff et al ${ }^{15}$ proposed that arterial remodelling acts to preserve levels of endothelial shear stress but, in his study, shear stress did not change with treatment despite the different effects on lumen diameter and carotid blood flow (CBF). Measurement of CBF suggested that $\mathrm{CC}$ was associated with a decrease in cerebrovascular resistance which is consistent with previous results which showed that ARBs act as cerebral vasodilators. ${ }^{32}$ In our study CBF was not measured.

The differences between these studies may relate to small sample sizes, the non-equivalence of BP reduction, or variable duration of therapy. The sample size of our study did not allow us to speculate. Further investigation will help us to elucidate these preliminary observations.

The total incidence of AEs was comparable between the two groups. However, a significantly greater number of AEs related to treatment was observed in the AML group compared with the CC group.

In conclusion, this study shows for the first time that $\mathrm{CC}$ and AML treatments can decrease CIMT in more than half of type 2 diabetic patients with hypertension. Even if this study was not placebo designed for ethical reasons, the present data strongly suggest that both drugs may alter the natural progression of CIMT in such patients, mainly as a result of BP lowering, the beneficial effect of $\mathrm{CC}$ in vascular remodeling deserves to be confirmed in a bigger sample of diabetic patients with or without hypertension. 


\section{Acknowledgments}

This study was supported by a grant from Laboratoires Takeda, Puteaux, France. We are indebted to all participating physicians, without whose collaboration this study would not have been possible. Andrea Lasserre from Medi.Axe, France, for medical writing services.

\section{Disclosures}

JMM is an employee of Laboratoires Takeda, Puteaux, France. The other authors have no conflicts of interest to disclose.

\section{References}

1. Wagenknecht LE, Zaccaro D, Espeland MA, Karter AJ, O'Leary DH, Haffner SM. Diabetes and progression of carotid atherosclerosis: the insulin resistance atherosclerosis study. Arterioscler Thromb Vasc Biol. 2003;23:1035-1041.

2. Chambless LE, Heiss G, Folsom AR, Rosamond W, Szklo M, Sharrett AR, et al. Association of coronary heart disease incidence with carotid arterial wall thickness and major risk factors: the Atherosclerosis Risk in Communities (ARIC) Study, 1987-1993. Am J Epidemiol. 1997;146:483-494.

3. O’Leary DH, Polak JF, Kronmal RA, Manolio TA, Burke GL, Wolfson SK Jr. Carotid artery intima and media thickness as a risk factor for myocardial infarction and stroke in older adults. Cardiovascular Health Study Collaborative Research Group. N Engl J Med. 1999;340:14-22.

4. Zanchetti A. Trials investigating the anti-atherosclerotic effects of antihypertensive drugs. J Hypertens. 1996; Suppl 14:S77-S80.

5. Wong M, Edelstein J, Wollman J, Bond MG. Ultrasonic-pathological comparison of the human arterial wall. Verification of intima-media thickness. Arterioscler Thromb. 1993;13:482-486.

6. Zanchetti A. Antiatherosclerotic effects of antihypertensive drugs: recent evidence and ongoing trials. Clin Exp Hypertens. 1996;18:489-499.

7. Elmfeldt D, George M, Hubner R, Olofsson B. Candesartan cilexetil, a new generation angiotensin II antagonist, provides dose dependent antihypertensive effect. J Hum Hypertens. 1997;11:S49-S53.

8. Pitt B, Byington RP, Furberg CD, Hunninghake DB, Mancini GB, Miller ME, et al; PREVENT Investigators. Effect of amlodipine on the progression of atherosclerosis and the occurrence of clinical events. Circulation. 2000;102:1503-1510.

9. Terpstra WF, May JF, Smit AJ, Graeff PA, Meyboom-de Jong B, Crijns HJ. Effects of amlodipine and lisinopril on intima-media thickness in previously untreated, elderly hypertensive patients (the ELVERA trial). J Hypertens. 2004;22:1309-1316.

10. Stanton AV, Chapman JN, Mayet J, Sever PS, Poulter NR, Hughes $\mathrm{AD}$, et al. Effects of blood pressure lowering with amlodipine or lisinopril on vascular structure of the common carotid artery. Clin Sci. 2001;101:455-464.

11. Valensi P, Baguet JP, Asmar R, Nisse-Durgeat S, Mallion JM. Effect of candesartan cilexetil on carotid intima-media thickness in hypertensive type 2 diabetic patients. MITEC study: design and baseline characteristics. Br J Diabetes Vasc Dis. 2007;7:18-24.

12. Guidelines Committee, 2003 European Society of HypertensionEuropean Society of Cardiology guidelines for the management of arterial hypertension. J Hypertens. 2003;21:1011-1053.

13. Baguet JP, Mallion JM, Moreau-Gaudry A, Noirclerc M, Peoc'h M, Siche JP. Relationships between cardiovascular remodeling and the pulse pressure in never treated hypertension. J Hum Hypertens. 2000;14:23-30.

14. Pignoli P, Tremoli E, Poli A, Oreste P, Paoletti R. Intimal plus medial thickness of the arterial wall: a direct measurement with ultrasound imaging. Circulation. 1986;7:1399-1406.
15. Ariff B, Zambanini A, Vamadeva S, Barratt D, Xu Y, Sever P, et al. Candesartan- and atenolol-based treatments induce different patterns of carotid artery and left ventricular remodeling in hypertension. Stroke. 2006;37:2381-2384.

16. Handa N, Matsumoto M, Maeda H, Hougaku H, Ogawa S, Fukunaga R, et al. Ultrasonic evaluation of early carotid atherosclerosis. Stroke. 1990;21:1567-1572.

17. Ludwig M, Koft K, Rucker W, Hunter AM. Diagnosis of very early arteriosclerotic vascular wall changes using duplex sonography. Klin Wocheuschr. 1989;67:442-446.

18. Hanefeld M, Chiasson JL, Koehler C, Henkel E, Schaper F, TemelkovaKurktschiev T. Arcabose slows progression of intima-media thickness of the carotid arteries in subjects with impaired glucose tolerance. Stroke. 2004;35:1073-1078.

19. Lonn EM, Yusuf S, Dzavik V, Doris C, Yi Q, Smith S, et al. Effects or ramipril and vitamin $\mathrm{E}$ on atherosclerosis: the study to evaluate carotid ultrasound changes in patients treated with ramipril and vitamin $\mathrm{E}$. Circulation. 2001;103:919-925.

20. Esposito K, Giugliano D, Nappo F, Marfella R. Regression of carotid atherosclerosis by control of postprandrial hyperglycemia in type 2 diabetes mellitus. Circulation. 2004;110:214-219.

21. Sidhu J, Kaposzta Z, Markus HS, Kaski JC. Effect of rosiglitazone on common carotid intima-media thickness progression in coronary artery disease patients without diabetes mellitus. Arterioscl Thromb Vasc Biol. 2004;24:930-934.

22. Simon A, Gariépy J, Moyse D, Levenson J. Differential effects of nifedipine and co-amilozide on the progression of early carotid wall changes. Circulation. 2001;103:2949-2954.

23. Zanchetti A, Bond MG, Hennning M, Neiss A, Mancia G, Dal Palu C, et al. Calcium antagonist lacidipine slows down progression of asymptomatic carotid atherosclerosis. Circulation. 2002;106:2422-2427.

24. Zanchetti A, Bond MG, Hennig M, Neiss A, Mancia G, Dal Palu C, et al. Risk factors associated with alterations in carotid intima-media thickness in hypertension: baseline data from the European Lacidipine Study on Atherosclerosis. J Hypertens. 1998;16:949-961.

25. Boutouyrie P, Bussy C, Hayoz D, Hengstler J, Dartois N, Laloux B, et al. Local pulse pressure and regression of arterial wall hypertrophy during long-term antihypertensive treatment. Circulation. 2000;101:2601-2606.

26. Mackinnon AD, Jerrard-Dunne P, Sitzer M, Buehler A, von Kegler S, Markus HS. Rates and determinants of site-specific progression of carotid artery intima-media thickness: the carotid atherosclerosis progression study. Stroke. 2004;35:2150-2154.

27. Schiffrin EL, Park JB, Intengan HD, Touyz RM. Correction of arterial structure and endothelial dysfunction in human essential hypertension by the angiotensin receptor antagonist losartan. Circulation. 2000;101:1653-1659.

28. Christensen KL, Mulvany MJ. Vasodilatation, not hypotension, improves resistance vessel design during treatment of essential hypertension: a literature survey. J Hypertens. 2001;19:1001-1006.

29. Ludwig M, Stapff M, Ribeiro A, Fritschka E, Tholl U, Smith RD, et al. Comparison of the effects of losartan and atenolol on common carotid artery intima-media thickness in patients with hypertension: results of 2 year, double blind, randomized, controlled study. Clin Ther. 2002;24:1175-1193.

30. Olsen MH, Wachtell K, Neland K, Bella JN, Rokkedal J, DigePetersen $\mathrm{H}$, et al. Losartan but not atenolol reduce carotid artery hypertrophy in essential hypertension. A LIFE substudy. Blood Press. 2005; 14:177-183.

31. Benetos A, Gautier S, Lafleche A, Topouchian J, Frangin G, Girerd X, et al. Blockade of Angiotensin II type 1 receptors: effect on carotid and radial artery structure and function in hypertensive humans. $J$ Vasc Res. 2000;37:8-15.

32. Vraamark T, Waldemar G, Strandgaard S, Paulson OB. Angiotensin II receptor antagonist CV-11974 and cerebral blood flow autoregulation. J Hypertens. 1995;13:755-761. 
